Article

\title{
Morphological transformation of SDS aggregates in a deep eu- tectic solvent above the fracto-eutectogel to fluid transition tem- perature
}

\author{
Lauren Matthews ${ }^{1,2, \dagger}$, Sarah E. S. Michel 1, Sarah E. Rogers ${ }^{3}$, Paul Bartlett ${ }^{1}$, Andrew J. Johnson ${ }^{4}$, Robert Sochon ${ }^{4}$, \\ and Wuge H. Briscoe ${ }^{1, *}$ \\ 1 School of Chemistry, University of Bristol, Cantock's Close, Bristol, BS8 1TS, UK. \\ 2 Bristol Centre for Functional Nanomaterials, HH Wills Physics Laboratory, University of Bristol, Tyndall \\ Avenue, BS8 1TL, UK. \\ 3 ISIS Muon and Neutron Source, Rutherford Appleton Laboratory, Harwell Oxford, Didcot, OX11 0QX, UK. \\ 4 GlaxoSmithKline, St George's Avenue, Weybridge, KT13 0DE, UK. \\ * Correspondence: Wuge.Briscoe@bristol.ac.uk; Tel.: +44 (0) 1173318256 \\ + Current address: ESRF, The European Synchrotron, 38043 Grenoble, France.
}

\begin{abstract}
Understanding surfactant self-assembly in deep eutectic solvents (DES) is important to their potential use in industrial formulations. We have recently reported the formation of a fractoeutectogel comprising SDS fractal aggregates at a concentration as low as $1.6 \mathrm{wt} \%$ in glyceline (a DES comprising glycerol and choline chloride) at room temperature. The building units of the fractals consisted of multilayers of self-assembled SDS lamellae arranged in a dendritic pattern. Here we report that this fractal phase transitions into a fluid phase above a critical gelation temperature, $T_{\mathrm{CG}} \sim 45^{\circ} \mathrm{C}$, evident from polarized light microscopy (PLM) observations. Small-angle neutron scattering (SANS) reveals that this phase transition is underpinned by the nanoscopic morphological transformation of the SDS lamellae into cylindrical micelles at $T>T_{\mathrm{GC}}$. Fitting SANS profiles confirms that the morphology of the micelles is SDS-concentration (CSDS) dependent at $T>T_{\mathrm{GC}}$ : cylindrical at $\operatorname{csDS}>0.6 \mathrm{wt} \%$ and spherical at $\operatorname{csDs}=0.6 \mathrm{wt} \%$. At $\operatorname{csDs}<0.6 \mathrm{wt} \%$, only isotropic scattering was observed in the SANS profiles. Such SDS self-assembly behaviors contrast with those we have previously observed in glycerol, which we attribute to the presence of ions (i.e. choline chloride) in glyceline. Our findings have general implications to surfactant self-assembly in DES, solvents that are rich in hydrogen bonding and ions.
\end{abstract}

Keywords: Deep eutectic solvents (DES), glyceline, surfactant mesophases, nonaqueous H-bonding solvents, small-angle neutron scattering (SANS), self-assembly, cylindrical micelles, spherical micelles, dendrites, fractals.

\section{Introduction}

Deep eutectic solvents (DES) have attracted considerable attention in recent years [1$4]$. With low volatility and green credentials, they are good solvents for a range of organic and inorganic species [5,6], making them desirable in applications such as electroplating [7-10] and formulations for pharmaceuticals [2] and personal care products [4, 11]. DES also have relevance in biological processes, e.g. biotransformations [12, 13].

Surfactant self-assembly in DES, relevant to many of these processes, has been investigated [14-17], with the formation of micelles of different structures above a critical micelle concentration (CMC) reported. Also as recently reported [11, 18, 19], the tendency for surfactants to form elongated aggregates in DES and glycerol can lead to gelation - at low surfactant concentrations at room temperature (RT) - due to 3D aggregates pervading 
the volume. This opens up new possibilities for their application, e.g. as delivery vectors, to explore the enhanced viscosity.

Gelation in DES (i.e. eutectogel formation) has been reported with 1,3:2,4-dibenzylidene-d-sorbitol (DBS) in reline, a DES comprising choline chloride and urea [19]. The structure of the gel was nano-fibrillar in nature, with the entanglement of these fibres causing the gelation. We have recently observed the formation of feather-like fractal aggregates at RT in glyceline [11], a mixture of choline chloride and glycerol in the 1:2 eutectic molar ratio, at SDS concentrations as low as $\operatorname{csDs}=0.1 \mathrm{wt} \%(\sim 1 \mathrm{CMC})$. At sufficient number densities (i.e. above a critical gelation concentration cсGC $>1.9 \mathrm{wt} \%$ ), the prevalence of the dendritic fractals led to the formation a colloidal gel, which we have termed the fracto-eutectogel.

However, the effect of temperature on gel phases in DES has been relatively under studied. Such knowledge is important to understanding the mechanisms of surfactant self-assembly in DES and also to the stability and manipulation of eutectogels in practical applications. We have previously noted that, at $T>\sim 45^{\circ} \mathrm{C}$, self-assembly and self-organisation of SDS in glyceline could be significantly affected, leading to the transition from the fracto-eutectogel phase to a fluid phase [11]. However, the nanoscopic structure underlying such a macroscopic phase transition was not clear. It has been reported in a related system that, at $T>\sim 45^{\circ} \mathrm{C}$, an SDS-in-glycerol gel underwent a lamellar gel-to-fluid transition, with the SDS aggregates transformed from lamellae, via an intermediate hexagonal phase, to cylindrical micelle solutions $[18,20]$.

We note that the effect of temperature on the morphology of surfactant mesophases is well reported in aqueous media [21-23]. For instance, a family of lysine-based surfactants formed a range of tubule structures below their Krafft points $\left(T_{\mathrm{k}}=25-49^{\circ} \mathrm{C}\right.$ depending on surfactant architecture); however, a tubule-to-vesicle transition was observed as the temperature was increased [24]. Of specific relevance to gelation, the transition between a lamellar liquid-crystalline phase and an $\alpha$-gel phase was observed at $T \sim 40{ }^{\circ} \mathrm{C}$ as a ternary gemini surfactant (synthesised from oleic acid), 1-tetradecanol, and water system was cooled [25].

Here, we report a detailed structural study of morphological transformations in the dendritic SDS aggregates that accompany the fracto-eutectogel-to-fluid transition in glyceline in the temperature range $25-70^{\circ} \mathrm{C}$. The phase was probed microscopically using polarised light microscopy (PLM) and the nanoscopic structure was studied using smallangle neutron scattering (SANS). At temperatures below the critical gelation temperature $\left(T_{\mathrm{GC}} \sim 45^{\circ} \mathrm{C}\right.$ ), the SANS profiles of SDS aggregates in the fracto-eutectogel could be well described by the lamellar paracrystal stack model or the mass fractal model. Above TGC, a micellar phase was formed, similar to that observed in SDS aqueous systems at room temperature [26, 27]. At $70{ }^{\circ} \mathrm{C}$, spherical SDS micelles were observed at $0.6 \mathrm{wt} \%$ SDS concentration (CSDS), which transformed to cylindrical micelles as CSDs was increased to $1.2 \mathrm{wt} \%$. Such varied aggregate morphologies highlight the differences in self-assembled SDS structures in glyceline, as compared with other nonaqueous solvents and with water. This demonstrates distinct molecular interactions in DES - rich in hydrogen bonds and ions as the driving force for molecular self-assembly, which is not currently fully understood.

\section{Results and Discussion}

\subsection{The effect of temperature on the microscopic structure of the surfactant mesophase}

Polarised light microscopy (PLM) revealed an anisotropic mesophase at the room temperature (RT) for samples with SDS concentrations cSDS $>1.9 \mathrm{wt} \%$ in glyceline, consisting of anisotropic fractal-like aggregates consistent with our previous observations [11], diminishing as the temperature was increased (at a rate $0.1{ }^{\circ} \mathrm{C} \mathrm{s}^{-1}$ ) and disappearing completely at $T=50-55^{\circ} \mathrm{C}$ (Error! Reference source not found.). This suggests the transformation of the aggregates to an isotropic structure and/or a structure smaller than the microscope resolution. Figure S1 shows the PLM images that captured the phase transition at $T=50-55^{\circ} \mathrm{C}$ at a finer temperature increment. 

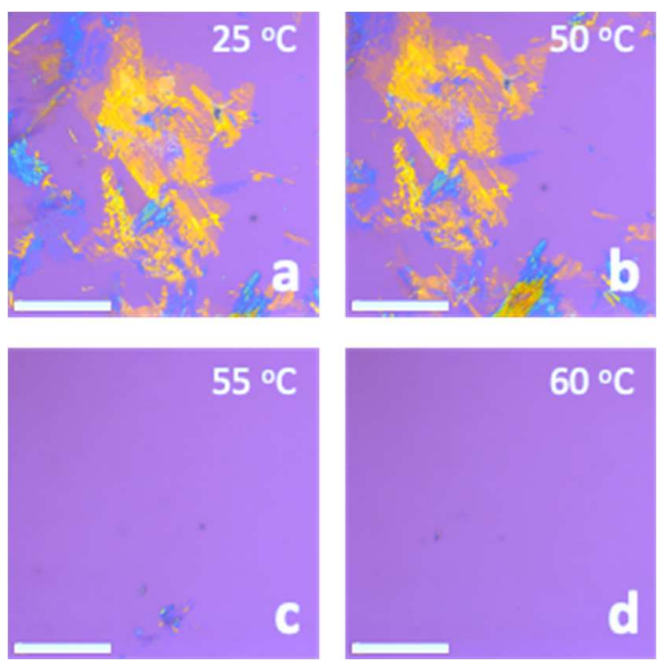

Figure 1. PLM images of $2.5 \mathrm{wt} \%$ SDS in glyceline at different temperatures as indicated, showing the disappearance of the fractal aggregates as the temperature was increased to $>\sim 50{ }^{\circ} \mathrm{C}$. Images were taken at $4 \times$ magnification with a $530 \mathrm{~nm}$ first order waveplate, and all scale bars represent $100 \mu \mathrm{m}$.

\subsection{Micellar nanostructure from SANS}

Figure 2a shows the SANS profiles for $h$-SDS ( $\operatorname{csDs}=5.3 \mathrm{wt} \%$; $40.7 \mathrm{CMC}$ ) in $d$-glyceline in the temperature range $T=25-70^{\circ} \mathrm{C}$, with the fitting parameters for the different models used list in Table 1-3 and modes described in section 4.4 below. Visual and microscopic observations confirmed that a gel (termed a fracto-eutectogel) transitioned into a fluid phase at $T>T_{\mathrm{CG}} \sim 40-50^{\circ} \mathrm{C}$ [11]. Of the micellar models trialed, a core-shell-cylinder form factor, $\mathrm{P}(\boldsymbol{q})$, was found to best fit the data above the TcG without any structure factor, $\mathrm{S}(\boldsymbol{q})$, suggesting non-interacting cylinders in solution despite the anionic nature of SDS. This may be rationalized by the presence of choline and chloride ions $(\sim 33 \mathrm{wt} \% ; 6.84 \mathrm{M}$ choline chloride) which may effectively screen the interactions between the aggregates.

Table 1. Fitting parameters for the lamellar stack paracrystal model (cf. Figure 2c) used to simulate the data for $\operatorname{cSDS}=5.3 \mathrm{wt} \% h$-SDS in $d$-glyceline in the gel phase at 25,35 , and $40{ }^{\circ} \mathrm{C}$ (cf. Figure 2a): SDS bilayer thickness $t$, number of layers in the stack $n$ Layers, $d$-spacing, polydispersity of the $d$-spacing $\sigma \mathrm{d}$, scattering length density of SDS $\rho$ sDS, scattering length density of glyceline $\rho$ Gly, polydispersity of the SDS bilayer thickness $\sigma_{\mathrm{t}}$, and chi squared value $\chi^{2}$.

\begin{tabular}{cccc}
\hline Model Parameters & $25^{\circ} \mathrm{C}$ & $35{ }^{\circ} \mathrm{C}$ & $40{ }^{\circ} \mathrm{C}$ \\
\hline$t_{\mathrm{L}}(\AA)$ & 20.0 & 20.6 & 20.0 \\
$n$ Layers & 57.7 & 57.8 & 57.8 \\
$d$-Spacing $(\AA)$ & 20.4 & 20.4 & 20.8 \\
$\sigma_{\mathrm{d}}(\AA)$ & 0.013 & $\sim 0$ & 0.067 \\
$\rho_{\text {SDS }}\left(10^{-6} \AA^{-2}\right)$ & 0.40 & 0.44 & 0.35 \\
$\rho_{\text {Gly }}\left(10^{-6} \AA^{-2}\right)$ & 5.50 & 5.83 & 5.75 \\
$\sigma_{\mathrm{t}}$ & 1.0 & 1.0 & 1.0 \\
$\chi^{2}$ & 3.8 & 2.6 & 3.5 \\
\hline
\end{tabular}




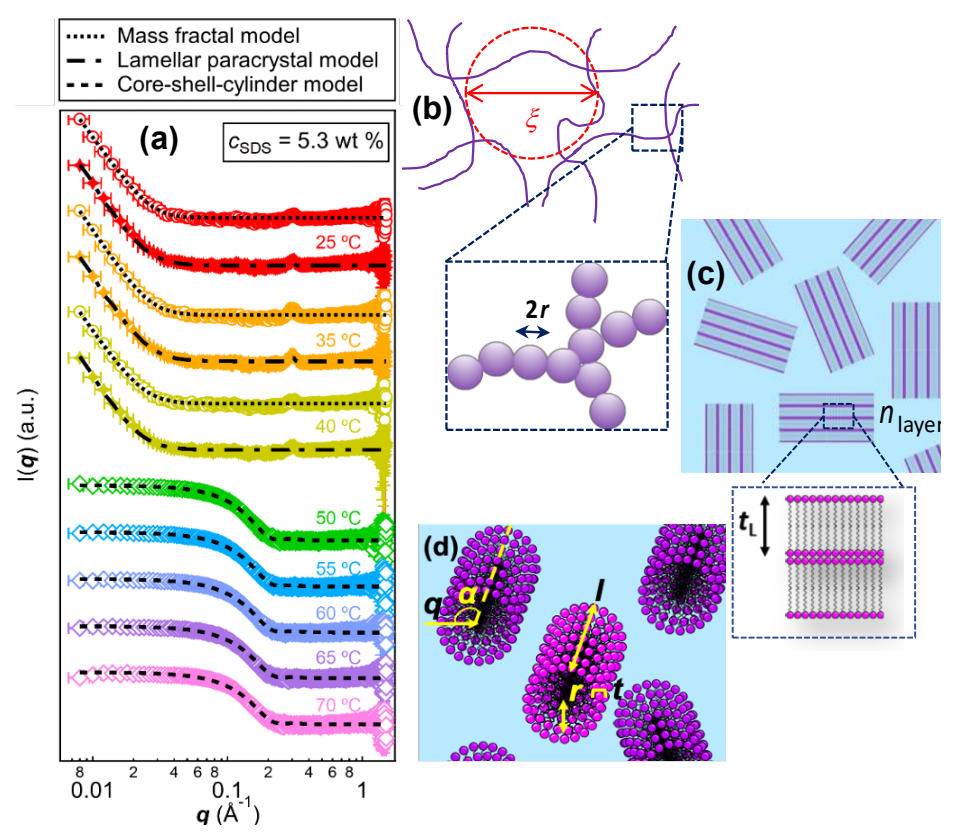

Figure 2. (a) SANS profiles for $5.3 \mathrm{wt} \% h$-SDS in $d$-glyceline at different temperatures $\left(25-70{ }^{\circ} \mathrm{C}\right)$, showing the transition from the fracto-eutectogel phase to the micellar phase at a critical gelation temperature, $T_{\mathrm{CG}} \sim 40-50{ }^{\circ} \mathrm{C}$. Fits to the profiles are indicated by black lines, and different lines are used to relate to the type of model used in the fit, shown in the legend: (b) the mass fractal model where $2 r=D$ is the building block diameter and $\xi$ the correlation length; (c) the lamellar stack paracrystal model with randomly oriented lamellar domains consisting of $n$ layers of bilayers of thickness $t_{\mathrm{L}}$, and (d) the core-shell cylinder model with cylinders of core radius $r$, shell thickness $t$, and length $l$. These models are described in section 4.4 below. The SANS profiles in (a) are offset on the vertical scale for clarity.

Table 2. Fitting parameters for the mass fractal model (cf. Figure $2 \mathrm{~b}$ ) used to simulate the data for 5.3 wt $\%$ h-SDS in $d$-glyceline at 25,35, and $40{ }^{\circ} \mathrm{C}$ (cf. Figure 2a): radius of the fractal aggregate $r$, fractal dimension $D_{\mathrm{m}}$, and chi squared value $\chi^{2}$.

\begin{tabular}{cccc}
\hline Model Parameters & $25^{\circ} \mathrm{C}$ & $35^{\circ} \mathrm{C}$ & $40^{\circ} \mathrm{C}$ \\
\hline$r(\AA)$ & 56.5 & 30.8 & 30.0 \\
$D_{\mathrm{m}}$ & 2.95 & 3.00 & 2.98 \\
$\chi^{2}$ & 3.4 & 3.1 & 3.7 \\
\hline
\end{tabular}

Table 3. Fitting parameters for the core-shell cylinder model (cf. Figure 2d) used to simulate the data for $5.3 \mathrm{wt} \% h$-SDS in $d$-glyceline in the fluid phase at 50, 55, 60, 65, and $70{ }^{\circ} \mathrm{C}$ (cf. Figure 2a): core radius $r$, shell thickness $t$, cylinder length $l$, scattering length density of the core $\rho_{\text {core, }}$ scattering length density of the shell $\rho_{\text {shell, }}$ scattering length density of glyceline $\rho_{\text {Gly, }}$, polydispersity of the core radius $\sigma_{\mathrm{r}}$, polydispersity of the shell thickness $\sigma_{\mathrm{t}}$, polydispersity of the cylinder length $\sigma_{\mathrm{l}}$, and chi squared value $\chi^{2}$.

\begin{tabular}{cccccc}
\hline Model Parameters & $50{ }^{\circ} \mathrm{C}$ & $55{ }^{\circ} \mathrm{C}$ & $60{ }^{\circ} \mathrm{C}$ & $65{ }^{\circ} \mathrm{C}$ & $70{ }^{\circ} \mathrm{C}$ \\
\hline$r(\AA)$ & 17.5 & 17.1 & 16.8 & 16.3 & 16.3 \\
$t(\AA)$ & 5.0 & 5.0 & 5.0 & 4.8 & 4.8 \\
$l(\AA)$ & 26.4 & 25.7 & 25.1 & 23.9 & 23.9 \\
$\rho_{\text {core }}\left(10^{-6} \AA^{-2}\right)$ & -0.31 & -0.42 & -0.40 & -0.38 & -0.38 \\
$\rho_{\text {shell }}\left(10^{-6} \AA^{-2}\right)$ & 4.98 & 5.03 & 4.95 & 4.80 & 4.80 \\
$\rho_{\text {Gly }}\left(10^{-6} \AA^{-2}\right)$ & 5.79 & 5.82 & 5.83 & 5.87 & 5.87 \\
$\sigma_{\mathrm{r}}$ & 0.05 & 0.05 & 0.05 & 0.05 & 0.05 \\
$\sigma_{\mathrm{t}}$ & 0.05 & 0.05 & 0.05 & 0.05 & 0.05 \\
$\sigma_{1}$ & 0.05 & 0.05 & 0.05 & 0.05 & 0.05 \\
\hline
\end{tabular}


As also reported in our previous work [11], ordered surfactant mesophases were present at $T<\sim 40-45{ }^{\circ} \mathrm{C}$ for samples at $\operatorname{csDs} \geq 1.2 \mathrm{wt} \%$ evident from a Bragg peak at $q \sim 0.30 \AA$ in the SANS profile (Figure $2 \mathrm{a}$ ). The profiles could be fitted to a lamellar paracrystal model with a lamellar spacing $d \sim 2 \pi / q \sim 20.9 \AA$. The Bragg peaks were indicative of the presence of the domains of solvated SDS lamellar crystals constituting the fractal dendrites[11].

Figure 3a shows the SANS profiles at different SDS concentrations, csDs, at a higher temperature $\left(T=70^{\circ} \mathrm{C}\right)$. Fitting the data to various models shows a transition in the form factor, $\mathrm{F}(q)$, from isotropic scattering ( $\operatorname{csDs}=0.1 \mathrm{wt} \%$ and $0.3 \mathrm{wt} \%)$, to a core-shell sphere model $\left(\operatorname{csDs}=0.6 \mathrm{wt} \%\right.$, apparent CMC at $\left.70{ }^{\circ} \mathrm{C}\right)$, and then to a core-shell cylinder model $(\operatorname{csDS}>1.2 \mathrm{wt} \%)$ as $\operatorname{csDs}$ was increased. The fitting parameters are listed in Table 4 and 5. This contrasts with the observation of fractal aggregates for all $\operatorname{csDs}$ at $T=25^{\circ} \mathrm{C}$, where a critical fractal aggregate concentration ( $C \mathrm{CFC})$ can be defined, below which no fractal aggregates were observed. The observations of $\mathrm{CCFC}=0.1 \mathrm{wt} \%$ at $25{ }^{\circ} \mathrm{C}$ and the $\mathrm{CMC}=0.6$ wt $\%$ at $70{ }^{\circ} \mathrm{C}$ point to the different self-assembly behaviors at the RT and the elevated temperature. The formation of crystallites was observed at $\mathrm{CCFC}=0.1 \mathrm{wt} \%$, however, the $\mathrm{CMC}$ at $70^{\circ} \mathrm{C}, \mathrm{CMC}_{70}=0.6 \mathrm{wt} \%$. This can be rationalized by considering an effective Krafft temperature of SDS in glyceline, below which crystallization is favored and thus the formation of the fractal aggregates with solvated lamellae; and above $T_{K}$ in the fluid phase, SDS self-assembly dominates.

The dimension of the cylindrical micelles varied as a function of both $T$ and csDs (Figure 4) where for all $\operatorname{csDs}$ both the radius, $r$, and length, $l$, of the cylinders decreased with increasing temperature. The length of cylindrical aggregates decreasing with increasing $T$ has been observed in other systems, attributed to the greater thermal energy $\left(k_{\mathrm{b}} T\right)$ which would encourage breakup of longer aggregates [28]. The decreasing cylinder radius, $r$, with increasing $T$ has also been observed previously and is explained by the increased motions and fluidity of the hydrophobic chains at higher $T$, reducing steric effects and allowing for better packing [29]. The radius of the cylindrical micelles increased with decreasing concentration in agreement with theoretical descriptions, where the micellar radius would initially increase with concentration, but then decrease due to the increasing number of micelles [30]. The cylinder length here increased with CSDS, due to the increased number of SDS molecules incorporated in individual micelles at high surfactant concentrations. 


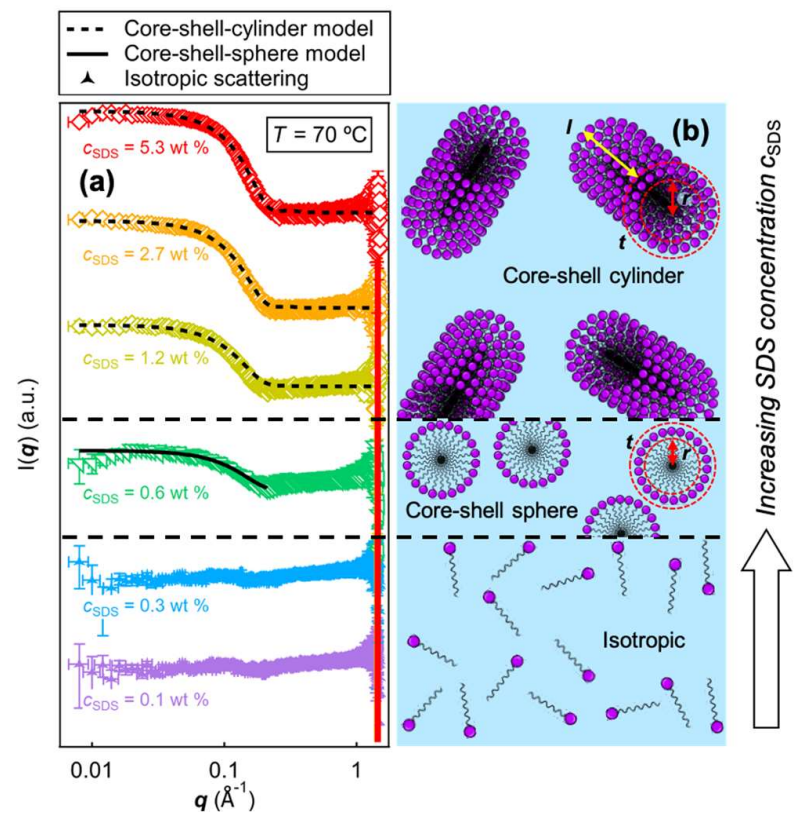

Figure 3. (a) SANS profiles for $h$-SDS in $d$-glyceline at varying surfactant concentrations, $\operatorname{csDS}$, at $T=$ $70{ }^{\circ} \mathrm{C}$. Also shown are fits to different models used (cf. Figure $3 \mathrm{~b}$ ). The profiles are offset on the vertical scale for clarity. (b) Schematic representation of the models used as the SDS concentration CsDs increased: the core-shell cylinder model (top) the core-shell sphere model (middle), and isotropic scattering (bottom).

Table 4. Fitting parameters for the core-shell cylinder model used to simulate the data for 1.2, 2.7, and $5.3 \mathrm{wt} \% h$-SDS in $d$-glyceline at $70{ }^{\circ} \mathrm{C}$ (cf. Figure 3a): core radius $r$, shell thickness $t$, cylinder

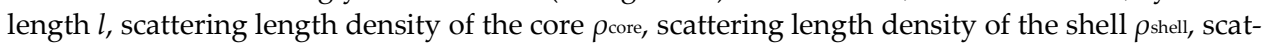
tering length density of glyceline $\rho$ Gly, polydispersity of the core radius $\sigma r$, polydispersity of the shell thickness $\sigma_{\mathrm{t}}$, polydispersity of the cylinder length $\sigma_{\mathrm{l}}$, and chi squared value $\chi^{2}$.

\begin{tabular}{cccc}
\hline Model Parameters & $1.2 \mathrm{wt} \%$ & $2.7 \mathrm{wt} \%$ & $5.3 \mathrm{wt} \%$ \\
\hline$r(\AA)$ & 16.8 & 16.9 & 16.3 \\
$t(\AA)$ & 5.0 & 5.0 & 4.8 \\
$l(\AA)$ & 23.9 & 23.6 & 21.1 \\
$\rho_{\text {core }}\left(10^{-6} \AA^{-2}\right)$ & -0.38 & -0.34 & -0.39 \\
$\rho_{\text {shell }}\left(10^{-6} \AA^{-2}\right)$ & 4.80 & 4.80 & 4.80 \\
$\rho_{\text {Gly }}\left(10^{-6} \AA^{-2}\right)$ & 5.87 & 5.86 & 5.88 \\
$\sigma_{\mathrm{r}}$ & 0.05 & 0.05 & 0.05 \\
$\sigma_{\mathrm{t}}$ & 0.05 & 0.05 & 0.05 \\
$\sigma_{\mathrm{l}}$ & 0.05 & 0.05 & 0.05 \\
$\chi^{2}$ & 2.45 & 2.07 & 4.31 \\
\hline
\end{tabular}

Table 5. Fitting parameters for the core-shell sphere model used to simulate the data for $0.6 \mathrm{wt} \%$ $h$-SDS in $d$-glyceline at $70{ }^{\circ} \mathrm{C}$ (cf. Figure 3a): core radius $r$, shell thickness $t$, scattering length density of the core $\rho_{\text {core, }}$ scattering length density of the shell $\rho_{\text {shell, scattering length density of }}$ glyceline $\rho_{\text {Gly }}$, polydispersity of the core radius $\sigma_{\mathrm{r}}$, polydispersity of the shell thickness $\sigma_{\mathrm{t}}$, and chi squared value $\chi^{2}$.

\begin{tabular}{cc}
\hline Model Parameters & $0.6 \mathrm{wt} \%$ \\
\hline$r(\AA)$ & 16.4 \\
$t(\AA)$ & 5.0 \\
$\rho_{\text {core }}\left(10^{-6} \AA^{-2}\right)$ & -0.30 \\
$\rho_{\text {shell }}\left(10^{-6} \AA^{-2}\right)$ & 5.12 \\
\hline
\end{tabular}




\begin{tabular}{cc}
$\rho_{\text {Gly }}\left(10^{-6} \AA^{-2}\right)$ & 5.74 \\
$\sigma_{\mathrm{r}}$ & 0.05 \\
$\sigma_{\mathrm{t}}$ & 0.05 \\
$\chi^{2}$ & 1.35 \\
\hline
\end{tabular}

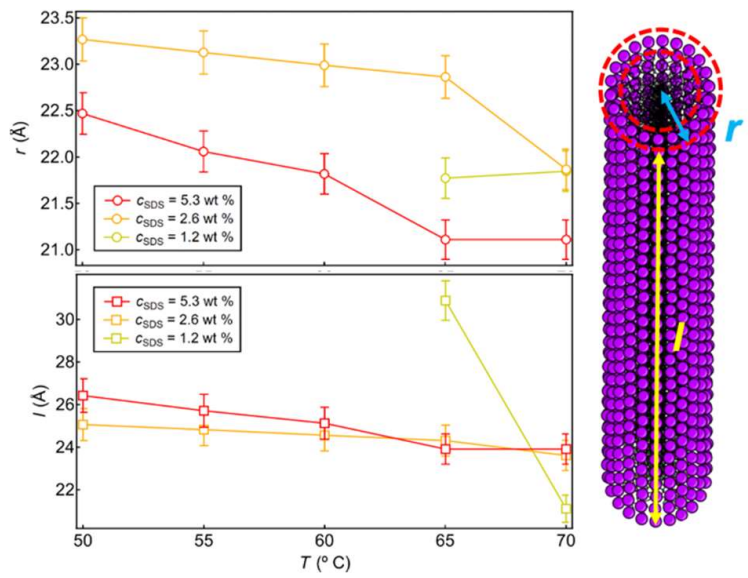

Figure 4. The effect of temperature, $T$, and $\operatorname{csss}$ on the radius, $r$, (top) and length, $l$, (bottom) of cylindrical aggregates formed in the glyceline bulk taken from fitting to SANS profiles, for $5.3 \mathrm{wt}$ $\%$ (red), $2.7 \mathrm{wt} \%$ (orange), and $1.2 \mathrm{wt} \%$ (yellow) wt \% SDS in glyceline. Schematic representation of the core-shell cylinder model shown (right).

\section{Further Discussions and Concluding Remarks}

We have probed the morphological changes that occurred upon the SDS-in-glyceline (a DES) fracto-eutectogel-to-fluid transition. This transition manifested in the disappearance of fractal dendrites as observed with PLM, and the change in the SANS profiles. The SANS profiles at the room temperature were fitted to a mass fractal model consistent with the dendritic aggregates observed microscopically with PLM. As discussed in [11], rheological measurements in this system confirmed a fracto-eutectogel consisting of globular aggregates in solution, akin to a physical colloidal gel with interpenetrating dendritic fractal aggregates. Here we show that above the critical gelation temperature, $T_{\mathrm{GC}} \sim 45^{\circ} \mathrm{C}$, a gel-to-fluid transition was observed, which was characterized nano-structurally with SANS. The fluid phase was shown to comprise a globular micellar fluid phase, where the observed morphology was concentration dependent, evident from the SANS fitting analyses.

SANS analyses also revealed morphological transformations of SDS micelles in the fluid phase at $T \sim 70{ }^{\circ} \mathrm{C}$. Whilst isotropic scattering was observed below an apparent $\mathrm{CMC}$, cSDs $<0.6 \mathrm{wt} \%$, spherical micelles were observed at $\operatorname{csDs} \sim 0.6 \mathrm{wt} \%$, and cylindrical micelles at $\operatorname{csDS}>0.6 \mathrm{wt} \%$. Morphological transformations of micelles have also been reported in aqueous SDS systems due to the addition of salt, rather than change in the SDS concentration. For instance, spherical or ellipsoidal micelles $[26,27,31]$ have been observed at RT at similar $\operatorname{csDs}(\operatorname{csDs} \sim 6.0 \mathrm{wt} \%)$, although micelles persisted through to very high SDS concentrations - up to $40 \mathrm{wt} \%$ at $70{ }^{\circ} \mathrm{C}$ in water). Furthermore, upon addition of sodium chloride $\left(c_{\text {salt }}=1-2 \mathrm{M}\right)$, flexible wormlike SDS micelles are observed in water [32-34]. The effect of salt addition on the SDS micelle shape in water could be well explained by the packing parameter: screening of the electrostatic headgroup repulsion would reduce the effective headgroup area, leading to a larger packing parameter $\left(C P P=V_{0} / a_{e} l\right)$ and aggregates of a smaller curvature, accounting for the sphere-to-cylinder transition. 
This highlights the differences of self-assembly in DES, where the formation of cylindrical aggregates without additional salt was observed in SDS self-assembly. This transition from gel to a fluid phase has been reported in an SDS-in-glycerol gel [18]. However, the lack of structure factor, $S(q)$, in the glyceline system (cf. Figure 3a), differs from that observed in glycerol [18] and also in DES systems as previously reported [14, 35]. Previous modelling of SANS profiles of ionic surfactants in reline and glyceline have included a hard sphere structure factor, $S(\boldsymbol{q})[15,35]$, pointing to interacting globular aggregates. The absence of an $S(\boldsymbol{q})$ here suggests that the globular aggregates were non-interacting, which is atypical for micelles consisting of ionic surfactants. The effective concentration of choline chloride in glyceline is $6.84 \mathrm{M}$, with a corresponding Debye length $\kappa^{-1} \sim 0.086 \mathrm{~nm}$. This suggests that the presence of choline and chloride ions in the glyceline bulk screens the micellar aggregates from each other very effectively. It has also been suggested that the choline ions could actively participate in the surfactant crystallization in forming the fractal aggregates at RT [11], encouraging dissociation of choline chloride.

The differences between the observations in glyceline and in glycerol [18] indicate that the self-assembly behavior is significantly influenced by the presence of choline chloride in glyceline which dissociates and screens interactions between SDS and between aggregates. Our previous results of SDS in glycerol showed a microfibrillar gel phase with lamellar nanostructure [18] at the room temperature, transitioning to a fluid phase consisting of cylindrical aggregates interacting via a Coulombic pair potential. The elongation of the SDS micelles observed with increasing CSDs in glyceline suggests that either the headgroup area decreases or the tail volume increases, to account for the reduction in curvature observed. Another explanation is related to the increasing number density of SDS micelles with increasing csDS. For anionic SDS micelles, this would result in pockets of negative charges within the bulk medium, which act to screen interactions among SDS micelles. The positive choline ions would associate with the negative micelles with the ability to bridge between micelles [11]. The length of the cylinders, relatively short compared to SDS-in-water cylindrical aggregates (wormlike micelles, $l>1000 \AA[36,37]$ ), is consistent with the choline ions bridging and linking $2-3$ spherical micelles in forming cylindrical micelles.

Our results here, in corroboration with our previous observations in glycerol [18] and in glyceline at RT [11], illustrate the self-assembly of surfactants in polar-nonaqueous polar DES media is complex. We are currently extending the work to investigate self-assembly of cationic surfactants and lipids in DES.

\section{Materials and Methods}

\subsection{Materials}

$h$-Sodium dodecyl sulfate (SDS) (Sigma-Aldrich, > 98.0 \%) was recrystallized three times from ethanol prior to use, and its purity was checked with ${ }^{1} \mathrm{H}$ NMR. $h$-Glycerol (Fisher Scientific, $>98.0 \%$ ) and $d$-Glycerol (Sigma-Aldrich, $>98.0 \%$ and $>98.0$ atom \% D) were used as received. $h$-Choline chloride (Sigma-Aldrich, $>98.0 \%$ ) and $d_{9}$-trimethyl-choline chloride (Sigma-Aldrich, $>98.0 \%$ and $>98.0$ atom \% D) were used as received. All glyceline-containing (both hydrogenated $(h-)$ and deuterated $(d-))$ phases and controls were kept sealed from moisture. $h$-/d-Glyceline was prepared by mixing $h$-/ $d$-choline chloride and $h$-/d-glycerol in a 1:2 molar ratio and leaving in a shaker incubator (Stuart SI505) for two hours ( $2 \mathrm{~h})$ at $550 \mathrm{RPM}$ at $60{ }^{\circ} \mathrm{C}$ until homogenous. The gel-like phase was prepared by adding a designated amount of $h$-SDS to $h$ - or $d$-glyceline, then incubating the mixture in the shaker incubator for at $550 \mathrm{RPM}$ at $60{ }^{\circ} \mathrm{C}$ for $2 \mathrm{~h}$ before equilibrating at room temperature overnight.

\subsection{Polarised light microscopy (PLM)}

PLM was carried out using an Olympus BX53-P microscope and a Nikon Eclipse E200 microscope, where the polarizers were crossed at $90^{\circ}$ with respect to each other and images were captured using Stream and PixeLINK® Capture OEM software, respectively. 4, 
10, 20, and $40 \times$ magnifications were used, and heating was facilitated by a Linkam stage. A $530 \mathrm{~nm}$ first order waveplate was placed into the optical patch to improve the clarity of image details in some cases.

\subsection{Small-angle neutron scattering (SANS)}

SANS data was obtained from samples contained in quartz cells with a $2 \mathrm{~mm}$ path length over a $0.5 \mathrm{~h}$ integration time on the LOQ small-angle diffractometer [38] at the ISIS Pulsed Neutron Source (STFC Rutherford Appleton Laboratory, Didcot, UK). LOQ utilizes neutrons with wavelengths $\lambda=2-10 \AA$ and the data was collected in the $q$ range of 0.008-1.6 $\AA^{-1}$. The raw scattering data were corrected for the detector efficiency, sample transmission, and background scattering and converted to scattering cross-section data ( $\partial \Sigma / \partial \Omega$ vs $q)$ using MantidPlot [39]. The data were then converted to an absolute scale $\left(\mathrm{cm}^{-}\right.$ $\left.{ }^{1}\right)$ using the scattering intensity from a standard sample (a solid blend of hydrogenous and perdeuterated polystyrene) in accordance with established procedures [40].

4.4 SANS Data Analysis

The SANS data at $25^{\circ} \mathrm{C}$ was fitted using two different models. The first was a lamellar paracrystal stack model (cf. Figure 2c), in which individual lamellae stacks in solution were treated as being independent of each other, with the SDS layer considered as a whole rather than separate headgroup layers and a tail layer [41-44]. The general scattering intensity for lamellar systems is described as

$$
I(\boldsymbol{q})=\frac{2 \pi V P(\boldsymbol{q}) S(\boldsymbol{q})}{d \boldsymbol{q}^{2}}
$$

where $V$ is the scattering volume, $P(q)$ the form factor that describes the shape of the particles or the phase present, $S(\boldsymbol{q})$ the structure factor that describes the interparticle interaction, and $d$ the lamellar spacing.

The second model used to fit data at $25{ }^{\circ} \mathrm{C}$ was a mass fractal model (cf. Figure $2 \mathrm{~b}$ ) describing the scattering from fractal-like aggregates in solution [45]

where the form factor is

$$
I(\boldsymbol{q})=P(\boldsymbol{q}) S(\boldsymbol{q})
$$

$$
P(\boldsymbol{q})=F(\boldsymbol{q} r)^{2}
$$

and the structure factor is

$$
S(\boldsymbol{q})=\frac{\Gamma\left(D_{m}-1\right) \zeta^{D_{m}-1}}{\left[1+(\boldsymbol{q} \zeta)^{2}\right]^{\frac{\left(D_{m}-1\right)}{2}}} \frac{\sin \left[\left(D_{m}-1\right) \tan ^{-1}(\boldsymbol{q} \zeta)\right]}{\boldsymbol{q}}
$$

where $r$ is the radius of the building block, $\zeta$ the correlation length, and $D_{\mathrm{m}}$ the mass fractal dimension.

The SANS data at $70{ }^{\circ} \mathrm{C}$ was fitted using two different form factors, $P(q)$, in SasView, in a core-shell-cylinder model (cf. Figure 3b) and a core-shell sphere model (cf. Figure 3b), respectively. The general scattering intensity for a core-shell-cylinder is [46-48]

$$
P(\boldsymbol{q})=\frac{1}{V_{s}} F^{2}(\boldsymbol{q}, \alpha) \cdot \sin \alpha
$$

where 


$$
\begin{array}{r}
F(\boldsymbol{q}, \alpha)=\left(\rho_{c}-\rho_{s}\right) V_{c} \frac{\sin \left(\boldsymbol{q} \frac{1}{2} l \cos \alpha\right)}{\boldsymbol{q} \frac{1}{2} l \cos \alpha} \frac{2 J_{1}(\boldsymbol{q} r \sin \alpha)}{\boldsymbol{q} r \sin \alpha}+ \\
\quad\left(\rho_{s}-\rho_{\text {solv }}\right) V_{s} \frac{\sin \left(\boldsymbol{q}\left(\frac{1}{2} l+t\right) \cos \alpha\right)}{\boldsymbol{q}\left(\frac{1}{2} l+t\right) \cos \alpha} \frac{2 J_{1}(\boldsymbol{q}(r+t) \sin \alpha)}{\boldsymbol{q}(r+t) \sin \alpha}
\end{array}
$$

and

$$
V_{s}=\pi(r+t)^{2}(l+2 t)
$$

Here, $\alpha$ is the angle between $q$ and the cylinder axis, $V_{\mathrm{s}}$ is the total volume, $V_{\mathrm{c}}$ is the core volume, $l$ is the core length, $r$ is the core radius, $t$ is the shell thickness, $\rho_{\mathrm{c}}$ is the core SLD, $\rho_{\mathrm{s}}$ is the shell SLD, $\rho_{\text {solv }}$ is the solvent SLD, and $J_{1}$ is the first order Bessel function.

The general scattering intensity for a core-shell-sphere model is [46]

$$
P(\boldsymbol{q})=\frac{F^{2}(\boldsymbol{q})}{V}
$$

where

$$
F(\boldsymbol{q})=\frac{3}{V_{s}}\left[V_{c}\left(\rho_{c}-\rho_{s}\right) \frac{\sin \left(\boldsymbol{q} r_{c}\right)-\boldsymbol{q} r_{c} \cos \left(\boldsymbol{q} r_{c}\right)}{\left(\boldsymbol{q} r_{c}\right)^{3}}+V_{s}\left(\rho_{s}-\rho_{\mathrm{solv}}\right) \frac{\sin \left(\boldsymbol{q} r_{s}\right)-\boldsymbol{q} r_{s} \cos \left(\boldsymbol{q} r_{s}\right)}{\left(\boldsymbol{q} r_{s}\right)^{3}}\right]
$$

where $V_{\mathrm{s}}$ is the total scattering volume of the object, $V_{\mathrm{c}}$ the scattering volume of the core, $\rho_{\mathrm{c}}$ the scattering length density (SLD) of the core, $\rho_{\mathrm{s}}$ the SLD of the shell, $\rho_{\text {solv }}$ the SLD of the solvent, $r_{\mathrm{c}}$ the radius of the core, and $r_{\mathrm{s}}$ the sum of the radius of the core and the thickness of the shell.

Supplementary Materials: The following are available online at www.mdpi.com/xxx/s1; Figure S1: PLM images of $2.5 \mathrm{wt} \%$ SDS in glyceline between $T=50-55^{\circ} \mathrm{C}$ at a finer temperature increment step; Figure S2: PLM images of $2.5 \mathrm{wt} \%$ SDS in glyceline at different temperatures, showing the disappearance of the fractal aggregates with elevated temperature; Figure S3: Fitted SANS data for $5.3 \mathrm{wt} \%$ SDS in glyceline at $70^{\circ} \mathrm{C}$ using different models; Figure S4: Refined fitted SANS data for $5.3 \mathrm{wt} \% \mathrm{SDS}$ in glyceline at $70^{\circ} \mathrm{C}$ using a spherical and cylindrical model; Figure S5: Refined fitted SANS data for $0.6 \mathrm{wt} \% \mathrm{SDS}$ in glyceline at $70{ }^{\circ} \mathrm{C}$ using a spherical and cylindrical model; Figure S6: Fitted SANS profile for $0.6 \mathrm{wt} \% \mathrm{SDS}$ in glyceline at $70{ }^{\circ} \mathrm{C}$ using a spherical and cylindrical model, and a Hayter-MSA structure factor; Figure S7: SANS profiles for 0.1 and $0.3 \mathrm{wt} \% h$-SDS in $d$ glyceline at $T=343 \mathrm{~K}$ with attempted fits shown by solid black lines; Figure S8: SANS profiles for $2.7 \mathrm{wt} \% h$-SDS in $d$-glyceline at different temperatures; Figure S9: SANS profiles for $1.2 \mathrm{wt} \% h$-SDS in $d$-glyceline at different temperatures; Figure S10: SANS profiles for $0.6 \mathrm{wt} \% h$-SDS in $d$-glyceline at different temperatures; Figure S11: SANS profiles for $0.3 \mathrm{wt} \% h$-SDS in $d$-glyceline at different temperatures; Figure S18: SANS profiles for $0.1 \mathrm{wt} \% h$-SDS in $d$-glyceline at different temperatures. Table S1: Fitting parameters for the sphere model used to simulate the data for $5.3 \mathrm{wt} \% h$-SDS in $d$ glyceline at $70{ }^{\circ} \mathrm{C}$; Table S2: Fitting parameters for the ellipsoid model used to simulate the data for $5.3 \mathrm{wt} \% h$-SDS in $d$-glyceline at $70{ }^{\circ} \mathrm{C}$; Table S3: Fitting parameters for the cylinder model used to simulate the data for $5.3 \mathrm{wt} \% h$-SDS in $d$-glyceline at $70{ }^{\circ} \mathrm{C}$; Table S4: Fitting parameters for the parallepiped model used to simulate the data for $5.3 \mathrm{wt} \% h$-SDS in $d$-glyceline at $70{ }^{\circ} \mathrm{C}$; Table S5: Fitting parameters for the core-shell-sphere model used to simulate the data for $5.3 \mathrm{wt} \% h$-SDS in $d$-glyceline at $70{ }^{\circ} \mathrm{C}$; Table S6: Fitting parameters for the core-shell-cylinder model used to simulate the data for $5.3 \mathrm{wt} \% h$-SDS in $d$-glyceline at $70^{\circ} \mathrm{C}$; Table S7: Fitting parameters for the core-shellsphere model used to simulate the data for $0.6 \mathrm{wt} \% h$-SDS in $d$-glyceline at $70{ }^{\circ} \mathrm{C}$; Table S8: Fitting parameters for the core-shell-cylinder model used to simulate the data for $0.6 \mathrm{wt} \% h$-SDS in $d$ - 
glyceline at $70{ }^{\circ} \mathrm{C}$; Table S9: Fitting parameters for the core-shell-sphere and core-shell-cylinder model with Hayter-MSA $S(q)$ used to simulate the data for 0.6 wt $\% h$-SDS in $d$-glyceline at $70{ }^{\circ} \mathrm{C}$; Table S10: Fitting parameters for the paracrystalline lamellar stack model used to simulate the data for $2.7 \mathrm{wt} \% \mathrm{~h}$-SDS in $d$-glyceline at 25,35 , and $40{ }^{\circ} \mathrm{C}$; Table S11: Fitting parameters for the mass fractal model used to simulate the data for $2.7 \mathrm{wt} \% \mathrm{~h}$-SDS in $d$-glyceline at 25,35 , and $40{ }^{\circ} \mathrm{C}$; Table S12: Fitting parameters for the core-shell-cylinder model used to simulate the data for $2.7 \mathrm{wt} \% \mathrm{~h}$ SDS in $d$-glyceline at 50,55,60,65, and $70^{\circ} \mathrm{C}$; Table S13: Fitting parameters for the paracrystalline lamellar stack model used to simulate the data for $1.2 \mathrm{wt} \% h$-SDS in $d$-glyceline at 25 , and $40{ }^{\circ} \mathrm{C}$, Table S14: Fitting parameters for the mass fractal model used to simulate the data for $1.2 \mathrm{wt} \% \mathrm{~h}$ SDS in $d$-glyceline at 25 , and $40{ }^{\circ} \mathrm{C}$; Table S15: Fitting parameters for the core-shell-cylinder model used to simulate the data for $1.2 \mathrm{wt} \% \mathrm{~h}$-SDS in $d$-glyceline at 65 , and $70{ }^{\circ} \mathrm{C}$; Table S16: Fitting parameters for the mass fractal model used to simulate the data for $0.6 \mathrm{wt} \% h$-SDS in $d$-glyceline at 25 , and $40{ }^{\circ} \mathrm{C}$; Table S17: Fitting parameters for the core-shell-sphere model used to simulate the data for $0.6 \mathrm{wt} \% h$-SDS in $d$-glyceline at 65 , and $70{ }^{\circ} \mathrm{C}$; Table S18: Fitting parameters for the mass fractal model used to simulate the data for $0.3 \mathrm{wt} \% h$-SDS in $d$-glyceline at 25 , and $40{ }^{\circ} \mathrm{C}$; Table S19: Fitting parameters for the mass fractal model used to simulate the data for $0.3 \mathrm{wt} \% h$-SDS in $d$-glyceline at $25{ }^{\circ} \mathrm{C}$.

Author Contributions: Conceptualization, P.B., R.S. and W. H. B.; methodology, L. M., S. E. R., and W. H. B.; validation, L. M., and W. H. B.; formal analysis, L. M., and W. H. B.; investigation, L. M., S. E. S. M., and S. E. R.; resources, A. J. J., R. S., and W. H. B.; data curation, L. M., and S. E. S. M.; writing-original draft preparation, L. M.; writing-review and editing, W. H. B.; visualization, L. M.; supervision, P. B., A. J. J., R. S., and W. H. B.; project administration, A. J. J., R. S., and W. H. B.; funding acquisition, P. B., R. S., and W. H. B.

Funding: L.M. was supported by a studentship jointly funded through the Engineering and Physical Science Research Council (the Bristol Centre for Functional Nanomaterials (EPSRC, $\mathrm{EP} / \mathrm{L} 016648 / 1)$ ) and GlaxoSmithKline.

Data Availability Statement: SANS data is available online at https://data.isis.stfc.ac.uk/ under the DOI: 10.5286/ISIS.E.RB1810629.

Acknowledgments: We acknowledge the ISIS Muon and Neutron Source for the awarded beamtime under experiment number: RB1810629. We thank Z'. Przybyłowicz, and S. Ruscigno for their help with the SANS experiments. SasView, originally developed under NSF award DMR-0520547, contains code developed with funding from the European Union's Horizon 2020 research and innovation programme under the SINE2020 project, grant agreement No 654000.

Conflicts of Interest: The authors declare no conflicts of interest.

\section{References}

[1] A.P. Abbott, G. Capper, D.L. Davies, H.L. Munro, R.K. Rasheed, V. Tambyrajah, Preparation of novel, moisture-stable, Lewisacidic ionic liquids containing quaternary ammonium salts with functional side chains, Chem Commun (19) (2001) $2010-2011$.

[2] A.P. Abbott, E.I. Ahmed, K. Prasad, I.B. Qader, K.S. Ryder, Liquid pharmaceuticals formulation by eutectic formation, Fluid Phase Equilibr 448 (2017) 2-8.

[3] A.P. Abbott, J.C. Barron, K.S. Ryder, D. Wilson, Eutectic-Based Ionic Liquids with Metal-Containing Anions and Cations, Chemistry - A European Journal 13(22) (2007) 6495-6501.

[4] E.L. Smith, A.P. Abbott, K.S. Ryder, Deep Eutectic Solvents (DESs) and Their Applications, Chem Rev 114(21) (2014) $11060-11082$.

[5] R.D. Rogers, K.R. Seddon, Ionic liquids - Solvents of the future?, Science 302(5646) (2003) 792-793.

[6] K.R. Seddon, Ionic liquids - A taste of the future, Nat Mater 2(6) (2003) 363-365.

[7] A.P. Abbott, G. Capper, K.J. McKenzie, K.S. Ryder, Electrodeposition of zinc-tin alloys from deep eutectic solvents based on choline chloride, J Electroanal Chem 599(2) (2007) 288-294.

[8] A.P. Abbott, G. Capper, B.G. Swain, D.A. Wheeler, Electropolishing of stainless steel in an ionic liquid, T I Met Finish 83(1) (2005) 51-53. 
[9] A.P. Abbott, K. El Ttaib, G. Frisch, K.J. McKenzie, K.S. Ryder, Electrodeposition of copper composites from deep eutectic solvents based on choline chloride, Phys Chem Chem Phys 11(21) (2009) 4269-4277.

[10] A.P. Abbott, K. El Ttaib, G. Frisch, K.S. Ryder, D. Weston, The electrodeposition of silver composites using deep eutectic solvents, Phys Chem Chem Phys 14(7) (2012) 2443-2449.

[11] L. Matthews, S. Ruscigno, S.E. Rogers, P. Bartlett, A.J. Johnson, R. Sochon, W.H. Briscoe, Fracto-eutectogels: SDS fractal dendrites via counterion condensation in a deep eutectic solvent, Phys Chem Chem Phys 23(20) (2021) 11672-11683.

[12] J. Gorke, F. Srienc, R. Kazlauskas, Toward Advanced Ionic Liquids. Polar, Enzyme-friendly Solvents for Biocatalysis, Biotechnol Bioproc E 15(1) (2010) 40-53.

[13] J. Gorke, F. Srienc, R.J. Kazlauskas, ORGN 299-Deep eutectic solvents: Alternative reaction media for hydrolase-catalyzed reactions, Abstr Pap Am Chem S 236 (2008).

[14] T. Arnold, A.J. Jackson, A. Sanchez-Fernandez, D. Magnone, A.E. Terry, K.J. Edler, Surfactant Behavior of Sodium Dodecylsulfate in Deep Eutectic Solvent Choline Chloride/Urea, Langmuir 31(47) (2015) 12894-12902.

[15] A. Sanchez-Fernandez, T. Arnold, A.J. Jackson, S.L. Fussell, R.K. Heenan, R.A. Campbell, K.J. Edler, Micellization of alkyltrimethylammonium bromide surfactants in choline chloride: glycerol deep eutectic solvent, Phys Chem Chem Phys 18(48) (2016) 33240-33249.

[16] A. Sanchez-Fernandez, O.S. Hammond, A.J. Jackson, T. Arnold, J. Doutch, K.J. Edler, Surfactant-Solvent Interaction Effects on the Micellization of Cationic Surfactants in a Carboxylic Acid-Based Deep Eutectic Solvent, Langmuir 33(50) (2017) 14304-14314.

[17] A. Sanchez-Fernandez, G.L. Moody, L.C. Murfin, T. Arnold, A.J. Jackson, S.M. King, S.E. Lewis, K.J. Edler, Self-assembly and surface behaviour of pure and mixed zwitterionic amphiphiles in a deep eutectic solvent, Soft Matter 14(26) (2018) 5525-5536.

[18] L. Matthews, Ż. Przybyłowicz, S.E. Rogers, P. Bartlett, A.J. Johnson, R. Sochon, W.H. Briscoe, The curious case of SDS selfassembly in glycerol: Formation of a lamellar gel, J Colloid Interf Sci 572 (2020) 384-395.

[19] J. Ruiz-Olles, P. Slavik, N.K. Whitelaw, D.K. Smith, Self-Assembled Gels Formed in Deep Eutectic Solvents: Supramolecular Eutectogels with High Ionic Conductivity, Angew Chem Int Edit 58(13) (2019) 4173-4178.

[20] L. Matthews, M.C. Stevens, R. Schweins, P. Bartlett, A.J. Johnson, R. Sochon, W.H. Briscoe, Unexpected observation of an intermediate hexagonal phase upon fluid-to-gel transition: SDS self-assembly in glycerol, Colloid and Interface Science Communications 40 (2021) 100342.

[21] C. Han, Y. Guo, X. Chen, M. Yao, Y. Zhang, Q. Zhang, X. Wei, Phase behaviour and temperature-responsive properties of a gemini surfactant/Brij-30/water system, Soft Matter 13(6) (2017) 1171-1181.

[22] M.N. Islam, T. Kato, Temperature Dependence of the Surface Phase Behavior and Micelle Formation of Some Nonionic Surfactants, The Journal of Physical Chemistry B 107(4) (2003) 965-971.

[23] Y. Zhang, X. Chen, X. Liu, Temperature-Induced Reversible-Phase Transition in a Surfactant-Free Microemulsion, Langmuir 35(44) (2019) 14358-14363.

[24] I.S. Oliveira, M. Lo, M.J. Araújo, E.F. Marques, Temperature-responsive self-assembled nanostructures from lysine-based surfactants with high chain length asymmetry: from tubules and helical ribbons to micelles and vesicles, Soft Matter 15(18) (2019) 3700-3711.

[25] T. Sugahara, M. Akamatsu, H. Iwase, Y. Takamatsu, K. Sakai, H. Sakai, Structural Change of an $\alpha$-Gel ( $\alpha$-Form Hydrated Crystal) Induced by Temperature and Shear Flow in an Oleic Acid Based Gemini Surfactant System, Langmuir 36(17) (2020) $4695-4701$.

[26] P. Kekicheff, Phase-Diagram of Sodium Dodecyl-Sulfate Water-System .2. Complementary Isoplethal and Isothermal Phase Studies, J Colloid Interf Sci 131(1) (1989) 133-152.

[27] P. Kekicheff, B. Cabane, M. Rawiso, Macromolecules Dissolved in a Lamellar Lyotropic Mesophase, J Colloid Interf Sci 102(1) (1984) 51-70.

[28] L. Sepulveda, C. Gamboa, Effect of Temperature on the Viscosity of Cationic Micellar Solutions in the Presence of Added Salts, J Colloid Interf Sci 118(1) (1987) 87-90. 
[29] T.L. Lin, M.Y. Tseng, S.H. Chen, M.F. Roberts, Temperature-Dependence of the Growth of Diheptanoylphosphatidylcholine Micelles Studied by Small-Angle Neutron-Scattering, J Phys Chem-Us 94(18) (1990) 7239-7243.

[30] L.M. Bergström, Explaining the growth behavior of surfactant micelles, J Colloid Interf Sci 440 (2015) 109-118.

[31] P. Kekicheff, C. Grabiellemadelmont, M. Ollivon, Phase-Diagram of Sodium Dodecyl-Sulfate Water-System .1. A Calorimetric Study, J Colloid Interf Sci 131(1) (1989) 112-132.

[32] L.J. Magid, Z. Li, P.D. Butler, Flexibility of Elongated Sodium Dodecyl Sulfate Micelles in Aqueous Sodium Chloride: A SmallAngle Neutron Scattering Study, Langmuir 16(26) (2000) 10028-10036.

[33] M. Sammalkorpi, M. Karttunen, M. Haataja, Ionic Surfactant Aggregates in Saline Solutions: Sodium Dodecyl Sulfate (SDS) in the Presence of Excess Sodium Chloride $(\mathrm{NaCl})$ or Calcium Chloride $(\mathrm{CaCl} 2)$, The Journal of Physical Chemistry B 113(17) (2009) 5863-5870.

[34] Z. Chu, C.A. Dreiss, Y. Feng, Smart wormlike micelles, Chem Soc Rev 42(17) (2013) 7174-7203.

[35] A. Sanchez-Fernandez, K.J. Edler, T. Arnold, R.K. Heenan, L. Porcar, N.J. Terrill, A.E. Terry, A.J. Jackson, Micelle structure in a deep eutectic solvent: a small-angle scattering study, Phys Chem Chem Phys 18(20) (2016) 14063-73.

[36] M.A. da Silva, V. Calabrese, J. Schmitt, K.M.Z. Hossain, S.J. Bryant, N. Mahmoudi, J.L. Scott, K.J. Edler, Impact of wormlike micelles on nano and macroscopic structure of TEMPO-oxidized cellulose nanofibril hydrogels, Soft Matter 16(20) (2020) 4887-4896.

[37] G.V. Jensen, R. Lund, J. Gummel, T. Narayanan, J.S. Pedersen, Monitoring the Transition from Spherical to Polymer-like Surfactant Micelles Using Small-Angle X-Ray Scattering, Angewandte Chemie International Edition 53(43) (2014) 11524-11528.

[38] R.K. Heenan, J. Penfold, S.M. King, SANS at pulsed neutron sources: Present and future prospects, J Appl Crystallogr 30 (1997) 1140-1147.

[39] O. Arnold, J.C. Bilheux, J.M. Borreguero, A. Buts, S.I. Campbell, L. Chapon, M. Doucet, N. Draper, R. Ferraz Leal, M.A. Gigg, V.E. Lynch, A. Markvardsen, D.J. Mikkelson, R.L. Mikkelson, R. Miller, K. Palmen, P. Parker, G. Passos, T.G. Perring, P.F. Peterson, S. Ren, M.A. Reuter, A.T. Savici, J.W. Taylor, R.J. Taylor, R. Tolchenov, W. Zhou, J. Zikovsky, Mantid-Data analysis and visualization package for neutron scattering and $\mu$ SR experiments, Nuclear Instruments and Methods in Physics Research Section A: Accelerators, Spectrometers, Detectors and Associated Equipment 764 (2014) 156-166.

[40] G.D. Wignall, F.S. Bates, Absolute calibration of small-angle neutron scattering data, J Appl Crystallogr 20(1) (1987) $28-40$.

[41] J. Berghausen, J. Zipfel, P. Lindner, W. Richtering, Influence of water-soluble polymers on the shear-induced structure formation in lyotropic lamellar phases, J Phys Chem B 105(45) (2001) 11081-11088.

[42] M. Bergstrom, J.S. Pedersen, P. Schurtenberger, S.U. Egelhaaf, Small-angle neutron scattering (SANS) study of vesicles and lamellar sheets formed from mixtures of an anionic and a cationic surfactant, J Phys Chem B 103(45) (1999) 9888-9897.

[43] A. Caille, X-Ray Scattering by Smectic-a Crystals, Cr Acad Sci B Phys 274(14) (1972) 891-893.

[44] F. Nallet, R. Laversanne, D. Roux, Modeling X-Ray or Neutron-Scattering Spectra of Lyotropic Lamellar Phases - Interplay between Form and Structure Factors, J Phys Ii 3(4) (1993) 487-502.

[45] D.F.R. Mildner, P.L. Hall, Small-Angle Scattering from Porous Solids with Fractal Geometry, J Phys D Appl Phys 19(8) (1986) 1535-1545.

[46] A. Guinier, G. Fournet, Small-Angle Scattering of X-Rays, John Wiley \& Sons, Inc., New York, US, 1955.

[47] S.R. Kline, Reduction and analysis of SANS and USANS data using IGOR Pro, J Appl Crystallogr 39 (2006) 895-900.

[48] J.S. Pedersen, Analysis of small-angle scattering data from colloids and polymer solutions: modeling and least-squares fitting, Adv Colloid Interfac 70 (1997) 171-210. 